http://dx.doi.org/10.32929/2446-8355.2019v28n2p166-178

\title{
ASPECTOS PRODUTIVOS E EFICIÊNCIA NO USO DA ÁGUA EM CULTIVARES DE CANOLA IRRIGADA
}

\author{
Anderson Crestani Pereira ${ }^{1 *}$, Adroaldo Dias Robaina ${ }^{2}$, Marcia Xavier Peiter ${ }^{2}$, Marcos \\ Vinicius Loregian ${ }^{3}$, Jardel Henrique Kirchner ${ }^{4}$, Wellington Mezzomo ${ }^{5}$, Luis Humberto Bahú \\ Ben $^{6}$, Bruna Dalcin Pimenta ${ }^{5}$, Rogério Ricalde Torres ${ }^{7}$

\footnotetext{
${ }^{1}$ Engenheiro Agrônomo, Mestrando do Programa de Pós-Graduação em Engenharia Agrícola, da Universidade Federal de Santa Maria (UFSM), Campus Santa Maria - Departamento de Engenharia Rural, Centro de Ciências Rurais, Santa Maria - RS. *E-mail do autor correspondente: acrestanipereira@ gmail.com.

${ }^{2}$ Engenheiro(a) Agrônomo(a), Professor(a) Dr.(a), Centro de Ciências Rurais, Departamento de Engenharia Rural, Universidade Federal de Santa Maria (UFSM), Santa Maria - RS, Brasil.

${ }^{3}$ Engenheiro Agrônomo, Mestrando do Programa de Pós-Graduação em Agronomia, Universidade Federal de Santa Maria (UFSM), Santa Maria - RS, Brasil.

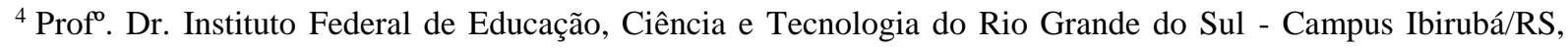
Brasil.

${ }^{5}$ Engenheiro(a) Agrônomo(a), M.Sc., Doutorando(a) do Programa de Pós-Graduação em Engenharia Agrícola, Universidade Federal de Santa Maria (UFSM), Santa Maria - RS, Brasil.

${ }^{6}$ Engenheiro Agrônomo, Doutor em Engenharia Agrícola, Universidade Federal de Santa Maria (UFSM), Santa Maria - RS, Brasil.

${ }^{7}$ Prof ${ }^{0}$. Dr. Instituto Federal de Educação, Ciência e Tecnologia do Rio Grande do Sul - Campus Vacaria/RS, Brasil.
}

Recebido: 23/11/2018; Aceito: 18/06/2019

RESUMO: A cultura da canola é a segunda oleaginosa mais produzida no mundo, possuindo importância no mercado mundial, principalmente pelo óleo utilizado no consumo humano e produção de biodiesel, sendo uma opção de renda ao produtor e também como alternativa de rotação de culturas. Com a irregular distribuição pluviométrica de algumas regiões, há necessidade do uso da irrigação para o suprimento hídrico adequado às plantas. Este trabalho objetiva avaliar a influência de lâminas de irrigação na produtividade, nos componentes de rendimento (número de siliquas por planta, número de grãos por siliqua e massa de mil grãos) e na eficiência do uso da água de duas cultivares de canola. O trabalho foi conduzido de junho a outubro de 2016, em delineamento experimental blocos ao acaso, com quatro repetições. $\mathrm{O}$ fator A foi constituído de 6 tratamentos de irrigação $(0 \%, 25 \%, 50 \%, 75 \%, 100 \%$ e $125 \%$ da $\mathrm{ET}_{\mathrm{o}}$ ), e o fator B por duas cultivares de canola (Hyola 61 e Diamond). A cultivar Diamond apresentou número de siliquas $26 \%$ superior à cultivar Hyola $61(\mathrm{P}<0,05)$. Com o uso da irrigação, houve incremento nos componentes de rendimento, na produtividade e aumento da eficiência do uso da água para ambas cultivares. A maior produtividade e eficiência do uso da água foram observadas na cultivar Diamond.

Palavras chave: Brassica napus L. Lâminas de irrigação. Componentes de rendimento.

\section{YIELD COMPONENTS AND WATER USE EFFICIENCY IN IRRIGATED CANOLA CULTIVARS.}


ABSTRACT: Canola cultivation is the second most produced oil seed in the world, having importance in the world market, mainly for the oil used in human consumption and biodiesel production, being an income option to the producer and also as an alternative crop rotation. With the irregular rainfall distribution of some regions, it is necessary to use the irrigation for adequate water supply to the plants. This work aims to evaluate the influence of irrigation depths on yield, yield components (number of siliquas per plant, number of grains per siliqua and mass of one thousand grains) and water use efficiency of two canola cultivars. The work was conducted from June to October 2016, in a randomized block design, with four replications. The factor A was composed of 6 irrigation treatments $(0 \%, 25 \%, 50 \%, 75 \%$, $100 \%$ and $125 \%$ of the ETo), and factor B by two canola cultivars (Hyola 61 and Diamond). The cultivar Diamond showed a number of siliquas $26 \%$ higher than the cultivar Hyola 61 (P $<0.05$ ). With the use of irrigation, there was an increase in yield components, productivity and an increase in the water use efficiency for both cultivars. The highest productivity and efficiency of water use were observed in the cultivar Diamond.

Key words: Brassica napus L. Irrigation depths. Yield components.

\section{INTRODUÇÃO}

A canola (Brassica napus L.) é uma oleaginosa importante para a agricultura pelo fato de ser rica em óleo vegetal comestível, além de ser uma importante fonte de energia renovável (TAN et al., 2009). A produção global de canola tem crescido rapidamente nos últimos 40 anos, passando da sexta para a segunda posição entre as oleaginosas cultivadas (USDA, 2017). No Brasil, a produção para o ano de 2017 foi estimada em 73,7 mil toneladas, sendo esta produção concentrada em apenas dois estados brasileiros: Rio Grande do Sul $(87,1 \%)$ e Paraná (12,9\%) (CARVALHO, 2017).

O cultivo dessa cultura constitui uma excelente alternativa na rotação de culturas, particularmente com a cultura do trigo no estado do Rio Grande do Sul (BATTISTI et al., 2013). Além disso, possui elevado valor agregado de comercialização, devido à boa qualidade nutricionais de seu óleo e também por ser uma alternativa para a produção de biodiesel, podendo ser uma opção de cultivo para os produtores do estado em relação à safra de outonoinverno.

A canola possui a particularidade de necessitar um período de vernalização, ou seja, temperaturas inferiores a $7^{\circ} \mathrm{C}$ por um período mínimo de oito semanas para que ocorra o florescimento (ANGELOTTI-MENDONÇA et al., 2016), fato este que a torna possível de ser cultivada no estado do Rio Grande do Sul.

Contudo, informações referentes ao manejo da cultura no estado, principalmente no que tange ao regime hídrico e suas interações com a produtividade, são escassas, motivando estudos que amparem diversos produtores, que tem aderido ao cultivo da canola e necessitam de informações em relação a sua necessidade hídrica para aumentar a eficiência do uso da água. 
Um dos principais entraves ao desenvolvimento regional da cultura tem sido o baixo rendimento médio de grãos, mesmo considerando as produtividades crescentes, com $1500 \mathrm{~kg}$ ha $^{-1}$ na safra 2015 e $1.514 \mathrm{~kg} \mathrm{ha}^{-1}$ na safra 2016 (CONAB, 2017). Para aumentar o rendimento de grãos é necessário estudar e identificar práticas de manejo, que visem o máximo aproveitamento do potencial genético da espécie (REGINATO, 2012), sendo a irrigação uma das principais estratégias possíveis. Neste sentido a determinação da eficiência no uso da água caracteriza-se como fator determinante para o correto dimensionamento e manejo da irrigação, sendo uma ferramenta indispensável na agricultura irrigada, evitando desperdícios do recurso hídrico disponível e diminuindo os custos da agricultura irrigada (ALI et al., 2007; KARAM et al., 2007).

O objetivo do trabalho foi avaliar a resposta produtiva, os componentes de rendimento e a eficiência do uso da água para produção das cultivares comerciais de canola Hyola 61 e Diamond submetidas à distintas lâminas de irrigação.

\section{MATERIAL E MÉTODOS}

O experimento foi conduzido na área experimental do Colégio Politécnico da Universidade Federal de Santa Maria (UFSM), Santa Maria, RS, (2931'S, 49 $19^{\prime}$ 'O e altitude média de 96 metros). De acordo com a classificação de Köppen, o clima da região é do tipo Cfa, subtropical úmido, com verões quentes e sem estação seca definida (HELDWEIN et al., 2009). O solo da área experimental é classificado como Argissolo Vermelho Distrófico Típico (STRECK et al., 2008).

As cultivares Diamond e Hyola 61 foram semeadas no dia 22 de junho de 2016, sob sistema de plantio direto, com uma densidade de 33 plantas por $\mathrm{m}^{2}$ e com espaçamento de $0,34 \mathrm{~m}$ entre linha. A adubação de base foi realizada com $300 \mathrm{~kg}$ do adubo com formulação 10-20-20.

A fertilização nitrogenada foi realizada de maneira fracionada, sendo baseada no teor de matéria orgânica no solo e na expectativa de produtividade da cultura $\left(2500 \mathrm{~kg} \mathrm{ha}^{-1}\right)$. Foi fornecido $30 \mathrm{~kg}$ de nitrogênio na adubação de base durante a semeadura, e, posteriormente em cobertura, foi realizada a segunda aplicação, utilizando uréia (45\% de N) na dose de $50 \mathrm{~kg}$ de $\mathrm{N} \mathrm{ha}^{-1}$, quando a cultura encontrava-se com quatro folhas verdadeiras formadas (COMISSÃO DE QUÍMICA E FERTILIDADE DO SOLO, 2016).

O experimento foi constituído de 48 parcelas, com dimensões de 4 x $6 \mathrm{~m}$, totalizando 24 $\mathrm{m}^{2}$ por parcela. Adotou-se o delineamento experimental em blocos ao acaso com quatro repetições, no qual o fator $A$ foi constituído de seis lâminas de irrigação suplementar $[0,25$, 50, 75, 100 e 125\% da evapotranspiração de referência (ETo)] e o fator B constituído de duas cultivares comerciais de canola (Diamond e Hyola 61).

O manejo de irrigação foi obtido através da ETo, com turno de rega fixo de sete dias, realizado quando a precipitação pluviométrica não suprisse a demanda evapotranspirativa da cultura. Para a determinação da ETo, foram utilizados dados meteorológicos de uma estação meteorológica automática do Instituto Nacional de Meteorologia (INMET), localizada na UFSM, a, aproximadamente, $2 \mathrm{~km}$ do local do experimento, onde diariamente foi monitorado 
a precipitação pluviométrica $(\mathrm{mm})$, temperatura $\left({ }^{\circ} \mathrm{C}\right)$, umidade relativa do ar $(\%)$, radiação solar $\left(\mathrm{w} \mathrm{m}^{-2}\right)$, fotoperíodo (horas) e velocidade do vento $\left(\mathrm{m} \mathrm{s}^{-1}\right)$, possibilitando, assim, o cálculo da evapotranspiração de referência pelo método de Penman-Monteith.

Foi considerada, também, a precipitação efetiva (PE), a qual foi calculada por meio do coeficiente de escoamento superficial "C", metodologia proposta por Millar (1978), que utiliza como parâmetros necessários as variáveis de classe textural do solo, declividade da área (\%) e cobertura vegetal. Diante disso, considerou-se como precipitação efetiva $70 \%$ da precipitação total ocorrida no intervalo entre as irrigações.

Para a aplicação da lâmina suplementar, foi utilizado um sistema de irrigação por aspersão convencional fixo. Os aspersores usados foram da marca AGROJET, modelo P5. O tempo de irrigação para aplicação da lâmina de irrigação foi determinado com base na taxa de aplicação de água do sistema, a qual foi obtida anteriormente a instalação do experimento, realizando o teste do Coeficiente de Uniformidade de Christiansen (CUC), sendo determinado o valor de $12,5 \mathrm{~mm} \mathrm{~h}^{-1}$. A diferenciação das lâminas de irrigação foi determinada por intermédio da variação do tempo de irrigação das distintas parcelas experimentais.

Para a determinação dos componentes de rendimento, os quais foram o número de siliquas por planta (NSP), número de grãos por siliqua (NGS) e massa de mil grãos (MMG), foram coletadas 10 plantas por parcela no fim do ciclo do cultivo, de ambas as cultivares.

Os grãos coletados foram secos em estufa durante 72 horas a uma temperatura de $65^{\circ} \mathrm{C}$. A massa de mil grãos foi determinada por meio de pesagem em balança de precisão, com exatidão de 0,1 g. Após, foram corrigidas para $10 \%$ de umidade em base úmida, segundo metodologia descrita nas regras de análises de sementes (BRASIL, 2009).

A produtividade da cultura foi determinada por meio da Equação 1, descrita a seguir.

$$
\text { PROD }=10 . \text { NP. NSP. NGS. MMG.1,1 }
$$

Em que:

PROD = produtividade de grãos $\left(\mathrm{kg} \mathrm{ha}^{-1}\right) ;$

$\mathrm{NP}=$ número de plantas por $\mathrm{m}^{2}$;

NSP = número de siliqua por planta;

NGS = número de grãos por siliqua; e

$\mathrm{MMG}=$ massa de mil grãos $(\mathrm{g})$.

Para a determinação da eficiência do uso da água para produtividade de grãos (EUA) foi utilizada a Equação 2:

$$
\mathrm{EUA}=\frac{\text { PROD }}{\text { TAA }}
$$

Em que:

EUA = eficiência do uso da água $\left(\mathrm{kg} \mathrm{m}^{-3}\right) ; \mathrm{e}$

TAA $=$ total de água aplicada $\left(\mathrm{m}^{3} \mathrm{ha}^{-1}\right)$.

As médias obtidas dos componentes de rendimento, produtividade e eficiência do uso da água foram submetidas à análise de variância, através do teste $\mathrm{F}$, com $5 \%$ de probabilidade de erro. Nos casos de diferenças estatisticamente significativas, realizou-se o teste Tukey para 
as variáveis qualitativas, e, regressão para as variáveis quantitativas, sendo ambas realizadas por intermédio do programa SISVAR (FERREIRA, 1998).

\section{RESULTADOS E DISCUSSÃO}

Durante o ciclo da canola, houve cinco irrigações e o volume total de chuva efetiva em todo experimento foi de $413 \mathrm{~mm}$, satisfazendo adequadamente as necessidades hídricas da cultura (Figura 1). Angelotti-Mendonça et al. (2016) definiram a necessidade hídrica adequada da canola no Rio Grande do Sul como, aproximadamente, $310 \mathrm{~mm}$ durante o ciclo. $\mathrm{O}$ valor encontrado nesse ensaio foi superior aos citados pelos autores. Entretanto, a distribuição foi irregular, com períodos de escassez e, portanto, houve suplementação hídrica através da irrigação quando necessário. Houve intervalos de até 14 dias sem precipitação. Tais períodos de escassez de chuvas são suficientes para influenciar negativamente os principais componentes de produtividade da canola avaliados neste estudo, conforme discutido a seguir.

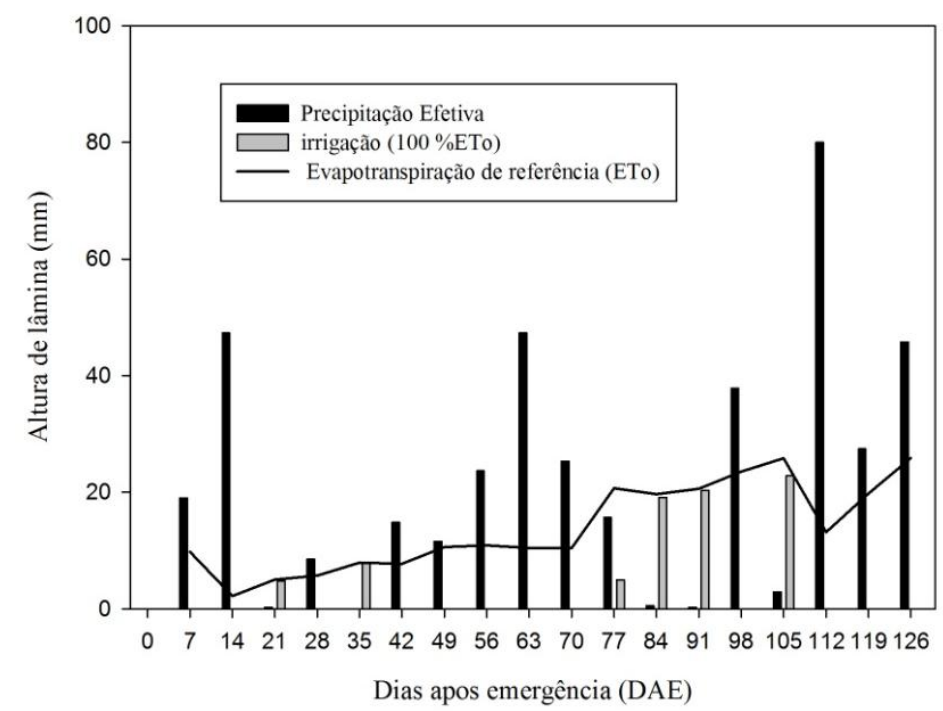

Figura 1. Consumo e aporte hídrico durante o ciclo da cultura. Consumption and water supply during the crop cycle.

Observou-se interação entre os tratamentos apenas para número de siliqua por planta $(\mathrm{P}<0,05)$. As demais variáveis não apresentaram interação estatisticamente significativa entre os fatores em estudo. Observou-se que as cultivares não diferiram estatisticamente entre si, para as variáveis número de siliquas por planta e massa de mil grãos. Por sua vez, para as variáveis número de grãos por siliqua, produtividade e eficiência do uso da água notaram-se melhores valores médios na cultivar Diamond, em comparação com a cultivar Hyola 61, tais resultados evidenciam que a cultivar Diamond é mais responsiva a irrigação suplementar.

Foi observado o valor de 202,45 siliquas por planta para cultivar Hyola 61 e, 204,75 siliqua por planta para a cultivar Diamond. Estes valores estão próximos com o descrito por Krüger et al. (2011) que trabalharam com distintas cultivares de canola no município de Augusto Pestana-RS, encontrando resultados similares para a cultivar Hyola 61.

Para o número de grãos por siliqua, a cultivar Hyola 61 apresentou menor valor em relação a cultivar Diamond, correspondendo a 17,95 e 22,62 grãos por siliqua, 
respectivamente. Isso ocorre, possivelmente, pela cultivar Diamond ter um potencial genético maior em comparação a cultivar Hyola 61. Estes valores estão dentro da faixa encontrada por Edwards \& Hertel (2011) que, em uma pesquisa compilando resultados de trabalhos publicados sobre a cultura da canola, citam que na maioria dos casos o número de grãos por siliqua oscila de 15 a 25 grãos por siliqua.

Tabela 1. Número de siliqua por planta (NSP), número de grãos por siliqua (NGS), massa de mil grãos (MMG), produtividade de grãos (PROD) e eficiência do uso da água (EUA) em resposta a cultivar e lâminas. Number of siliqua per plant (NSP), number of grains per siliqua (NGS), mass of one-thousand grains (MMG), grain yield (PROD) and water use efficiency in response to cultivar and depths.

\begin{tabular}{cccccc}
\hline \multirow{2}{*}{ Cultivares } & \multicolumn{5}{c}{ Variáveis } \\
\cline { 2 - 6 } & NSP & NGS & MMG (g) & PROD $\left(\mathrm{kg} \mathrm{ha}^{-1}\right)$ & EUA $\left(\mathrm{kg} \mathrm{m}^{3}\right)$ \\
\hline Hyola 61 & $202,45 \mathrm{a}$ & $17,95 \mathrm{~b}$ & $3,33 \mathrm{a}$ & $1881,83 \mathrm{~b}$ & $0,308 \mathrm{~b}$ \\
Diamond & $204,75 \mathrm{a}$ & $22,62 \mathrm{a}$ & $3,45 \mathrm{a}$ & $2090,25 \mathrm{a}$ & $0,342 \mathrm{a}$ \\
\hline CV $(\%)$ & 7,39 & 8,6 & 9,62 & 12,22 & 12,70 \\
\hline
\end{tabular}

Houve um incremento no NSP com os tratamentos de irrigação, demonstrando uma resposta positiva a irrigação suplementar ao longo do ciclo da cultura (Figura 2). O menor número de siliqua, para ambas cultivares, foi encontrado no tratamento testemunha, sendo de 162 siliquas para a cultivar Hyola 61 e 186 siliquas para a cultivar Diamond.

Esse menor valor de siliquas por planta pode estar atrelado à ocorrência de déficit hídrico ao longo do ciclo, o que pode ter limitado este componente. Esse fato corrobora com o que é relatado por Bilibio et al. (2011) que em experimento com distintos níveis de restrição hídrica na cultura da canola na Alemanha, citou uma redução do número de siliqua por planta de $22 \%$, quando a cultura foi submetida a $30 \%$ de restrição hídrica, chegando a uma redução de $26 \%$ na restrição de $60 \%$ da demanda hídrica da cultura.

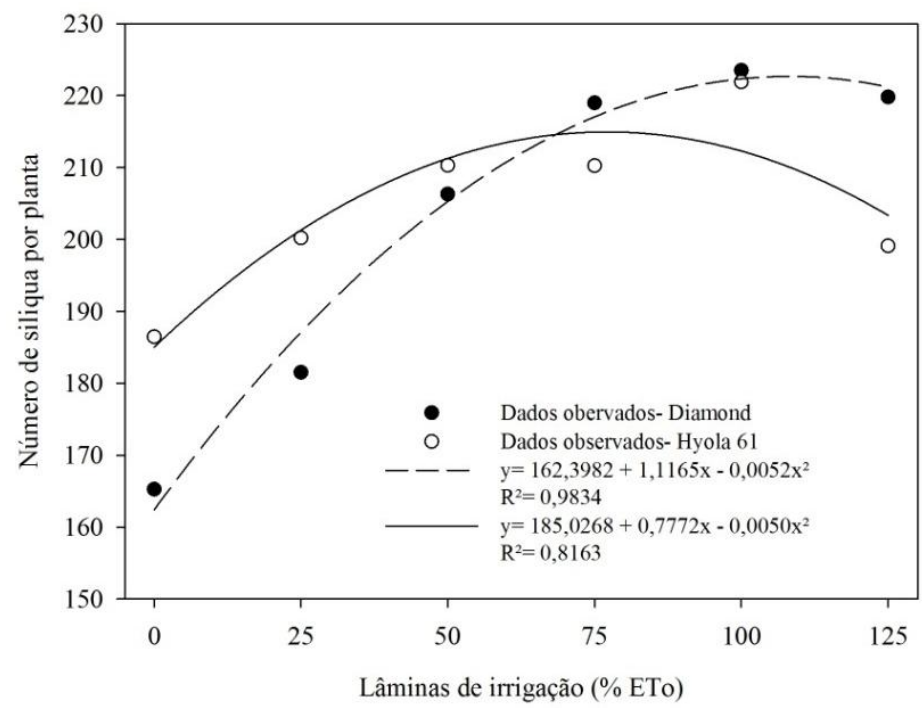

Figura 2. Número de siliqua por plantas observadas em respostas as distintas lâminas de irrigação. Number of siliqua per plants observed in response to different irrigation depths. 
Ademais, Dalmago et al. (2017), trabalhando com as cultivares Diamond e Hyola 61 submetidas a distintas porcentagens da capacidade de retenção de água no solo, obtiveram um valor médio de siliqua por planta $24 \%$ maior para a cultivar Diamond em comparação a Hyola 61. Os mesmos autores citam que, na mesma porcentagem de umidade do solo, a variação entre as cultivares para número de siliquas por planta é similar.

O comportamento do número de grãos por siliqua, em resposta as distintas lâminas de irrigação apresentam uma resposta positiva em ambas cultivares utilizadas (Figura 3). A máxima eficiência para número de grãos por siliqua foi observada no tratamento de $91 \%$ da ETo, chegando a 19,32 grãos por siliqua para a cultivar Hyola 61. Já para a cultivar Diamond, encontrou-se 22,58 grãos por siliqua no tratamento de $125 \%$ da ETo.

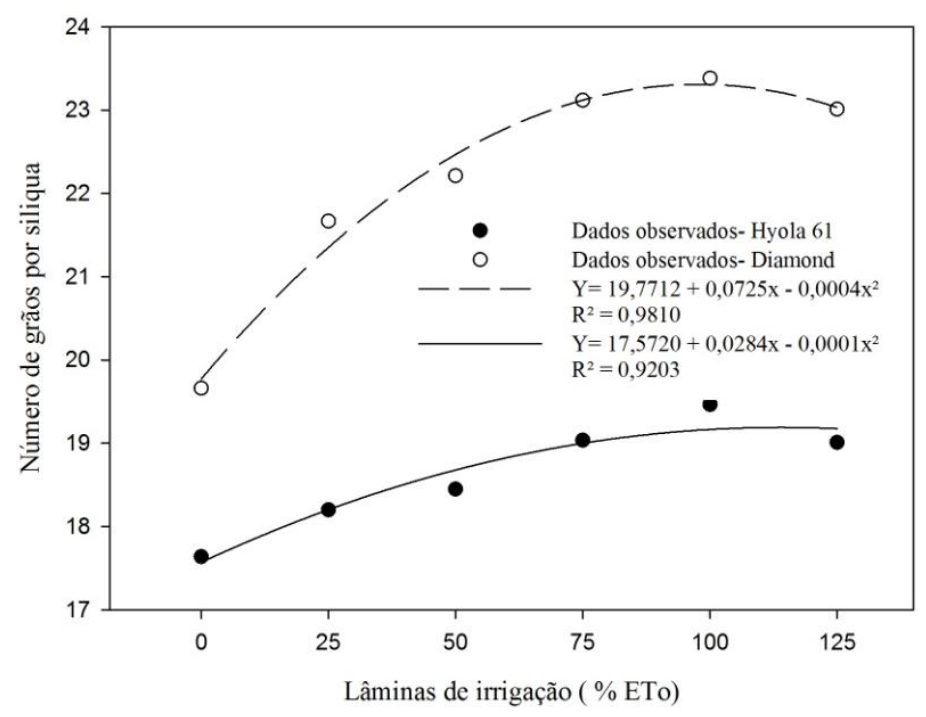

Figura 3. Efeito da irrigação sobre o número de grãos por siliqua. Effect of irrigation on the number of grains per siliqua.

Angelotti-Mendonça et al. (2016) afirmaram que condições de restrição hídrica no cultivo da canola influenciam negativamente o florescimento, a formação das síliquas e a formação de grãos, o que corrobora resultados encontrados nesse estudo, podendo, assim, justificar a oscilação presente no número de grãos por siliqua encontrados nos tratamentos.

Bandeira et al. (2013), pesquisando a resposta da canola em função da densidade de semeadura e espaçamento, relataram uma oscilação do número de grãos por siliqua entre dois anos agrícolas e atribuem essa variação ao distinto volume de precipitação, o que ocasionou uma diferença no aporte de fotoassimilados e a sua maior partição para as diferentes partes da planta.

As duas cultivares diferiram quanto a massa de mil grãos, sendo esse componente de extrema importância para a produtividade da cultura. A variação da massa de mil grãos para os distintos tratamentos está representada na Figura 4.

A massa de mil grãos aumentou para ambas cultivares com o aumento da lâmina de irrigação (Figura 4). A máxima eficiência foi observada no tratamento de $83 \%$ da ETo, obtendo-se 3,31 g para a cultivar Hyola 61. Por sua vez, para a cultivar Diamond, encontrouse 3,55 g no tratamento de $100 \%$ da ETo. 


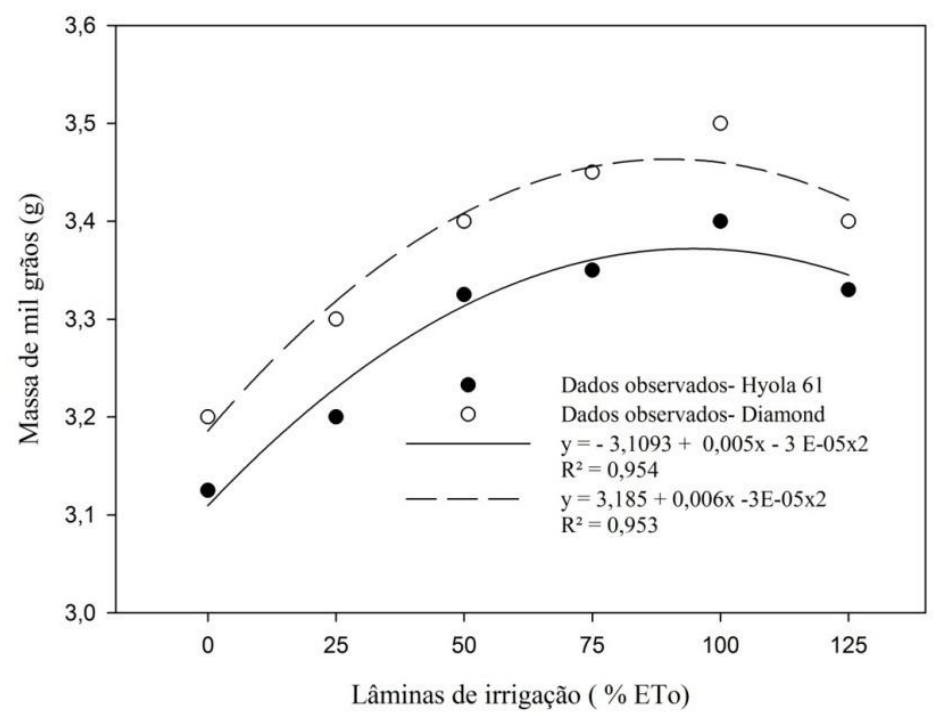

Figura 4. Massa de mil grãos em resposta a irrigação. Mass of thousand grains in response to irrigation.

O aumento da massa de mil grãos possivelmente está ligado ao aporte hídrico correto, uma vez que, segundo Angelotti-Mendonça et al. (2016) a falta de água compromete o enchimento dos grãos, diminuindo sua massa. Taylor et al. (1991) observaram diferença significativa do peso de 1000 grãos de canola no tratamento irrigado em comparação ao não irrigado. Bilibio (2010), trabalhando com a cultura da canola irrigada em Lavras-MG, utilizando distintas lâminas de reposição de água obtiveram em tratamentos irrigados um incremento de $14 \%$ na massa de 1000 grãos. Outros autores citam uma redução potencial da massa de mil grãos e da produção em função do estresse hídrico em torno de 34\% (TOHIDIMOGHADAMET et al., 2009; FARAJI et al., 2008). Em contraposto, Champolivier e Merrien (1996) não observaram diferença significativa do peso de 1000 grãos de canola exposta a tratamentos com déficit hídrico.

A produtividade aumentou para ambas as cultivares (Figura 5). A produtividade para a cultivar Hyola 61 oscilou de $1365,4 \mathrm{~kg} \mathrm{ha}^{-1}$ na testemunha a $2280 \mathrm{~kg} \mathrm{ha}^{-1}$ no tratamento de $100 \%$ ETo. Já para cultivar Diamond, os valores oscilam de $1679,75 \mathrm{~kg} \mathrm{ha}^{-1}$ na testemunha a $2326,75 \mathrm{~kg} \mathrm{ha}^{-1}$ no tratamento de $100 \%$ ETo. Essa variação de produtividade pode ser atrelada a déficit hídrico durante o ciclo de cultivo. Segundo Thomas (2013), a diminuição dos teores de água no solo reduz a expansão do sistema radicular e o crescimento da área foliar no período vegetativo. Já durante as fases de floração e enchimento de grãos, o déficit hídrico ocasiona aumento nos teores de ácidos graxos insaturados e redução dos saturados, diminuindo a qualidade do óleo (MOHAMMADI et al., ROKHZADL, A; 2012; DOGAN et al., 2011).

O incremento da produção de grãos nos tratamentos de irrigação pode ser explicado pela não ocorrência de déficit hídrico durante o ciclo de cultivo, principalmente durante a fase de floração. De acordo com Sanches et al. (2014), que pesquisaram a canola irrigada em Dourados-MS, concluíram que os componentes produtivos do híbrido Hyola 61 respondem significativamente à irrigação no estágio de floração plena, o que influenciou diretamente na produção final da cultura. Marjanovic-Jeromela et al. (2011), avaliando a cultura da canola em Maringa-PR, atribuíram variações da produtividade de inverno ao longo dos anos de 
cultivo à disponibilidade irregular de chuvas durante o ciclo da cultura, especialmente na fase de enchimento de grãos.

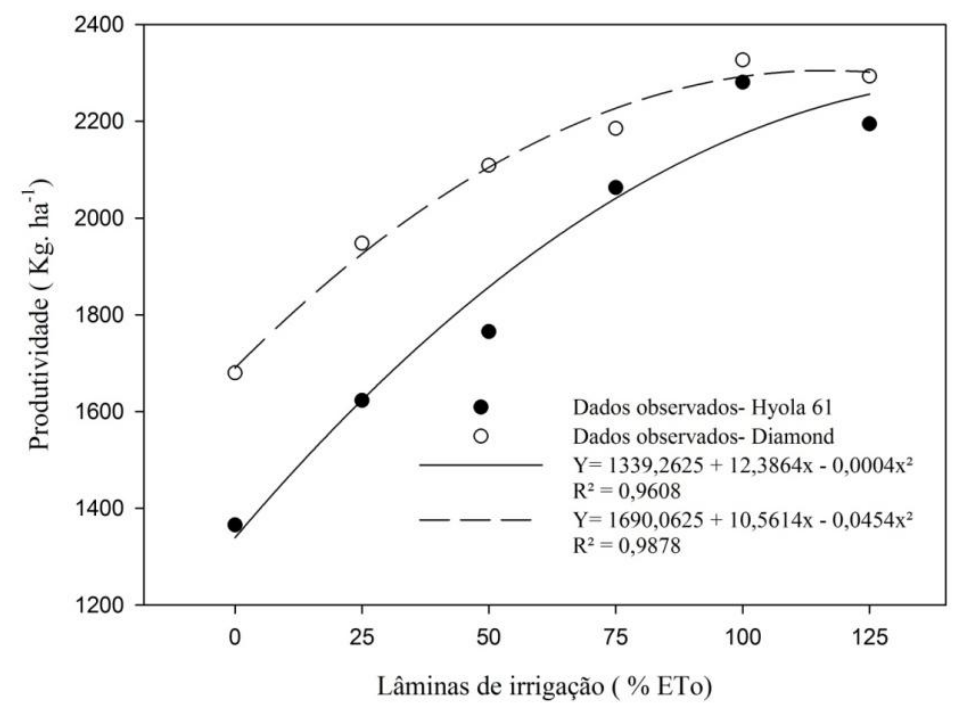

Figura 5. Produtividade observados nos distintos tratamentos. Productivity observed in the different treatments.

Nesse estudo, os máximos valores de eficiência técnica para produtividade foram obtidos nos tratamentos 125\% (2194 kg ha ${ }^{-1}$ ) e de $116 \%$ (2304 kg ha' $)$ para cultivares Hyola 61 e Diamond, respectivamente. Sob irrigação, alguns trabalhos demonstram que a produtividade de grãos de canola pode variar entre $2200 \mathrm{~kg} \mathrm{ha}^{-1}$ e $3200 \mathrm{~kg} \mathrm{ha}^{-1}$ (TOHIDIMOGHADAMET et al., 2009); no entanto, outros autores apontam para uma produtividade superior a $4000 \mathrm{~kg} \mathrm{ha}^{-1}$ (DOGAN et al., 2011; KAMKAR et al., 2011).

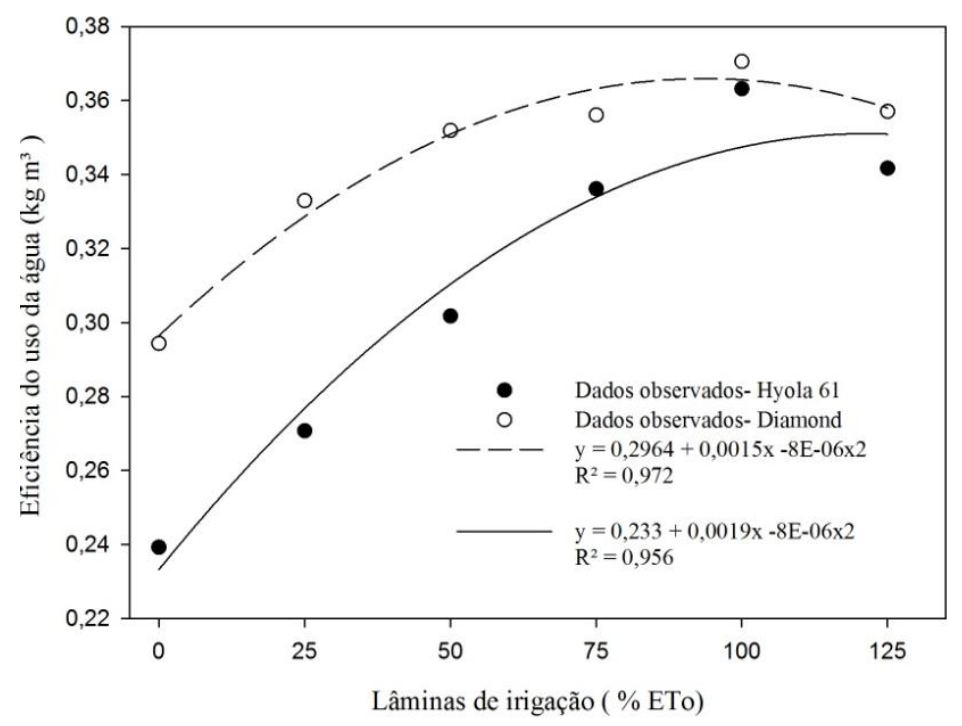

Figura 6. Eficiência de uso da água $\left(\mathrm{kg} \mathrm{m}^{-3}\right)$ nos distintos tratamentos. Efficiency of water use in the different treatments.

Na Figura 6 pode-se observar a eficiência de uso da água, que relaciona a produção comercial, que são os grãos, pela quantidade de água aplicada $\left(\mathrm{m}^{3}\right)$, nas distintas cultivares analisadas. Nota-se um aumento na eficiência do uso da água para ambas cultivares até os valores de $94 \%$ ETo $\left(0,3457 \mathrm{~kg} \mathrm{~m}^{-3}\right)$ e de $116 \%$ ETo $\left(0,3664 \mathrm{~kg} \mathrm{~m}^{-3}\right)$, nos quais obteve-se a 
máxima eficiência técnica, para as cultivares Hyola 61 e Diamond, respectivamente. O menor valor de EUA para a cultivar Hyola 61 foi de $0,24 \mathrm{~kg} \mathrm{~m}^{-3}$ e para Diamond foi de 0,29 $\mathrm{kg} \mathrm{m}^{-3}$, ambas para no tratamento testemunha.

O aumento da eficiência do uso da água com o uso da irrigação também foi constatado por Conceição et al. (2016), em que encontraram para a cultivar Hyola 61 valores de $0,27 \mathrm{~kg}$ $\mathrm{m}^{-3}$ para o tratamento testemunha e $0,55 \mathrm{~kg} \mathrm{~m}^{-3}$ para o tratamento de $100 \%$ ETc.

A maximização da eficiência do uso da água observado, em ambas cultivares, pode ser explicada pela disponibilidade adequada de água no solo, o que favorece os processos fisiológicos da planta, melhorando, assim, a expressão do potencial genético da cultivar uma vez que o fator água não será um limitante para a planta.

Segundo Angelotti-Mendonça et al. (2016), o manejo adequado da umidade do solo é responsável pelo crescimento do sistema radicular e da área foliar, favorecendo a retenção de folhas por mais tempo e acumulando maior quantidade de fotoassimilados, interferindo, dessa forma, nos componentes de rendimento da cultura da canola.

\section{CONCLUSÃO}

A irrigação proporcionou um acréscimo nos componentes de rendimento e aumento da produtividade obtida para ambas cultivares estudadas. Ocorreu um aumento na eficiência do uso da água na produção de grãos para ambas cultivares com a utilização dos tratamentos de irrigação testados. A cultivar Diamond apresentou maior produtividade e melhor eficiência do uso da água.

\section{REFERÊNCIAS BIBLIOGRÁFICAS}

ALI, M. H.; HOQUE, M. R.; HASSAN, A. A.; KHAIR, A. Effects of deficit irrigation on yield, water productivity and economic returns of wheat. Agricultural Water Management, Amsterdam, v. 92, n. 3, p.151-161, 2007.

ANGELOTTI-MENDONÇA, J.; RIBOLDI, L. B.; SOARES, C. D. F.; CASTRO, P. R. C.; KLUGE, R. A. Canola (Brassica napus L.). Piracicaba: ESALQ, 2016. 32 p. (Produtor rural, n. 61)

BANDEIRA, T. P., CHAVARRIA, G., \& TOMM, G. O. Desempenho agronômico de canola em diferentes espaçamentos entre linhas e densidades de plantas. Pesquisa Agropecuária Brasileira, Brasília, DF, v. 48, n. 10, p.1332-1341, 2013.

BATTISTI, R.; PILAU, F. G.; SCHWERZ, L.; SOMAVILLA, L.; TOMM, G. O. Dinâmica floral e abortamento de flores em híbridos de canola e mostarda castanha. Pesquisa Agropecuária Brasileira, Brasília, DF, v. 48, n. 2, p.174-181, 2013.

BRASIL. Ministério da Agricultura, Pecuária e Abastecimento. Secretaria de Defesa Agropecuária. Regras para análise de sementes. Brasília, DF: Mapa: ACS, 2009. 395 p. 
BILIBIO, C. G.; CARVALHO, J. A.; HENSEL, O.; RICHTER, U. Effect of different levels of water deficit on rapeseed (Brassica napus L.). Ciência e Agrotecnologia, Lavras, v. 35, n. 4, p.672-684, 2011.

CARvalHO, M. A. Canola. Brasília, DF: Conab, 2017. Disponível em http://www.conab.gov.br/OlalaCMS/uploads/arquivos/17_02_20_14_43_57_canola__conjuntura_mensal_-_janeiro_2017.pdf. Acesso em: 25 jun. 2018.

CHAMPOLIVIER, L.; MERRIEN, A. Effects of water stress applied at different growth stages to Brassica napus L. var. oleifera on yield, yield components and seed quality. European Journal of Agronomy, Paris, v. 5, n. 3/4, p.153-160, 1996.

COMISSÃO DE QUÍMICA E FERTILIDADE DO SOLO - CQFSRS/SC. Manual de adubação e calagem para os Estados do Rio Grande do Sul e de Santa Catarina. 11.ed. Porto Alegre: Sociedade Brasileira de Ciência do Solo, Núcleo Regional Sul, 2016. 400 p.

CONCEIÇÃO, C. G.; SOARES, F. C.; PARIZI, C. R. A; RODRIGUES, S. A.; PEITER, M. X.; ROBAINA, A. D.; GIRARDI, L. B.; BEN, L. H. Análise dos componentes do rendimento da canola irrigada na região Fronteira Oeste do Rio Grande do Sul. Tecnologia \& Ciência Agropecuária, João Pessoa, v. 10, n. 1, p.40-45, 2016.

DAlMAGO, G. A.; CUNHA, G. R.; GOUVEA, J. A.; BRUGNERA, L.; GREGOSKI, C. Respostas da canola à disponibilidade de água no solo. In: CONGRESSO BRASILEIRO DE AGROMETEOROLOGIA / SIMPÓSIO DE MUDANÇAS CLIMÁTICAS E DESERTIFICAÇÃO DO SEMIÁRIDO BRASILEIRO, 5 / 20, 2017, Juazeiro / Petrolina. Anais [...] Juazeiro: Univasf; Petrolina: Embrapa Semiárido, 2017. p. 1-5.

DOGAN, E.; COPUR, O.; KAHRAMAN, A.; KIRNAK, H.; GULDUR, M. E. Supplemental irrigation effect on canola yield components under semiarid climatic conditions. Agricultural Water Management, Amsterdam, v. 98, n. 9, p.1403-1408, 2011.

EDWARDS, J.; HERTEL, K. Canola growth and development. New South Wales: Department of Primary Industries, 2011. 87 p. Disponível em: http://www.dpi.nsw.gov.au/_data/assets/pdf_file/0004/516181/Procrop-canola-growthanddevelopment.pdf. Acesso em: 25 jun.2018.

FARAJI, A.; LATIFI, N.; SOLTANI, A.; RAD, A. H. S. Seed yield and water use efficiency of canola (Brassica napus L.) as affected by high temperature stress and supplemental irrigation. Agricultural Water Management, Amsterdam, v. 96, n. 1, p.132-140, 2008.

FERREIRA, D. F. Sisvar: sistema de análise de variância para dados balanceados. Lavras: UFLA, 1998. 19 p.HELDWEIN, A. B.; BURIOL, G. A.; STRECK, N. A. O clima de Santa Maria. Ciência \& Ambiente, Santa Maria, v.38, n. 1, p.43-58, 2009.

KAMKAR, B.; DANESHMAND, A. R.; GHOOSHCHI, F.; SHIRANIRAD, A. H.; SAFAHANILANGEOUDI, A. R. The effects of irrigation regimes and nitrogen rates on some agronomic traits of canola under semi arid environment. Agricultural Water Management, Amsterdam, v. 98, n. 6, p.1005-1012, 2011.

KARAM, F.; LAHOUD, R.; MASAAD, R.; KABALAN, R.; BREIDI, J.; CHALITA, C.; ROUPHAEL, Y. Evapotranspiration, seed yield and water use efficiency of drip irrigated 
sunflower under full and deficit irrigation conditions. Agricultural Water Management, Amsterdam, v. 90, n. 3, p.213-223, 2007.

KRUGER, C. A. M. B.; SIlvA, J. A. G.; MedeIROS, S. L. P.; DAlMAGO, G. A.; SARTORI, C. O.; SCHIAVO, J. Arranjo de plantas na expressão dos componentes da produtividade de grãos de canola. Pesquisa Agropecuária Brasileira, Brasília, DF, v. 46, n. 11, p.1448-1453, 2011.

MARJANOVIC-JEROMELA, A.; NAGAL, N; GVOZDANOVIC-VARGA, J.; HRISTOV, N; KONDIC-SPIKA, A.; VASIC, M; MARINKOVIC, R. Genótipo por interação ambiental para produção de sementes por planta em colza usando o modelo AMMI. Pesquisa Agropecuária Brasileira, Brasília, DF, v. 46, n. 2, p.174-181, 2011.

MILLAR, A. A. Drenagem de terras agrícolas: bases agronômicas. São Paulo: McGrawHill do Brasil, 1978. 276 p.

MOHAMMADI, K.; ROKHZADI, A. An integrated fertilization system of canola (Brassica napus L.) production under different crop rotations. Industrial Crops and Products, Amsterdam, v. 37, n. 1, p.264-269, 2012.

REGINATO, C. Rendimento da canola (Brassica napus L. var. Hyola 433) submetida a diferentes adubações foliares. Chapecó: [s. n.], 2012. 36 p.

SANCHES, A. C.; GOMES, E. P.; RAMOS, W. B. MAUAD, M.; SANTOS, S. D.; BISCARO, G. A. Produtividade da canola irrigada e doses de adubação nitrogenada. Revista Brasileira de Engenharia Agrícola e Ambiental, Campina Grande, v. 18, n. 7, p.688-693, 2014.

STRECK, E. V; KÄMPF, N; DALMOLIN, R. S. D; KLAMT, E; NASCIMENTO, P. C DO; SCHNEIDER, P; GIASSON, E; PINTO, L. F. S. Solos do Rio Grande do Sul. 2. ed. Porto Alegre: EMATER/RS - ASCAR, 2008. 222 p.

TAN, X.L.; KONG, F.M.; ZHANG, L.L.; LI, J.; CHEN, S.; QI, C.K. Cloning and analysis of hemoglobin gene in Cyanobacterium and transformation into Brassica napus (L.). Acta Agronomica Sinica, Zhongguancun Nan Dajie, v. 35, n. 1, p.66-70, 2009.

TAYLOR, A. J.; SMITH, C. J.; WILSON, I. B. Effect of irrigation and nitrogen fertilizer on yield, oil content, nitrogen accumulation and water use of canola (Brassica napus L.). Fertilizer Research, Copenhagen, v. 29, n. 3, p.249-260, 1991.

THOMAS, P. Canola grower's manual. Winnipeg: Canola Council of Canada, 2013. Disponível em: http://www.canolacouncil.org/crop-production/canola-grower's-manualcontents. Acesso em: 23 abr. 2018.

TOHIDI-MOGHADAM, H. R.; SHIRANI-RAD, A. H.; NOUR-MOHAMMADI, G.; HABIBI, D.; MODARRES-SANAVY, S. A. M.; MASHHADI-AKBAR-BOOJAR, M.; DOLATABADIAN, A. Response of six oilseed rape genotypes to water stress and hydrogel application. Pesquisa Agropecuária Tropical, Goiania, v. 39, n. 3, p.243-250, 2009.

USDA. United States Department of Agriculture. Production, supply and distribution. Disponível 
https://www.nass.usda.gov/Publications/Todays_Reports/reports/crop1117.pdf. Acesso em: 12 jun. 2018. 